\title{
MATRIZ DE AUTOEVALUACIÓN UNIVERSITARIA PARA LA CARRERA DE PSICOLOGÍA
}

\author{
Oswaldo Orellana Manrige *,D aphne Orellana Garca *
}

\begin{abstract}
RESUMEN
El documento constituye la segunda parte de una investigación exploratoria. Es la propuesta de una matriz para la autoevaluación universitaria, de la Facultad de Psicología de la Universidad Nacional Mayor de San Marcos.
\end{abstract}

Se precisa el carácter de la autoevaluación, los objetivos de mejora y la acreditación, así como las fases que transita el proceso de la autoevaluación, concluyendo con la matriz compuesta por veinte factores, haciendo una propuesta de indicadores y fuentes de verificación.

Palabras claves: Autoevaluación, Mejora, Metodología, Variable, Indicador, Fuente de verificación.

\begin{abstract}
The document constitutes the second part of an exploratory investigation. It is the proposal of a matrix for the university autoevaluación, from Faculty of Psychology University Nacional Mayor de San Marcos.

One needs the character the autoevaluación, the objectives of improvement and the accreditation, asi like the phases that the process of the autoevaluación journeys, concluding with the matrix composed by twenty factors, doing a proposal of indicators and sources of verification.
\end{abstract}

Keywords: Autoevaluación, Improvement, Methodology, Variable, Indicator, Source of verification.

\section{INTRODUCCIÓN}

La crisis que ha traído la globalización y las distintas expresiones del posmodernismo obligan buscar referentes para poder entender la institución universitaria. Uno de ellos es la calidad universitaria. Dentro de ella la excelencia académica, que debe ser la expresión más alta del cumplimiento de los objetivos para la cual la universidad fue creada, y por lo tanto la garantía pública de ser un espacio de producción de conocimientos para la formación de cuadros altamente calificados.

\footnotetext{
* Docente Principal. Facultad de Psicología de la UNMSM. E-mail: ovaorellana@ahoo.es

*Psicóloga-Investigadora Colaboradora. E-mail: daphneorellanagarcia@hoo.es
} 
En los ambientes universitarios las iniciativas para lograr la calidad han sido muchas, algunas centradas adecuadamente en términos de realizar una autoevaluación, plan de mejora y proyectarse a la acreditación, iniciándose con la sensibilización requerida y la capacitación, otras, reducidas a la voluntad de los deseos, y finalmente, unas terceras, trivializadas en aspectos secundarios, cubiertas bajo el lenguaje del «liderazgo», la «competitividad», con poco o nada de sentido público, pero con un marketing dispuesto a distorsionar ideológicamente el funcionamiento universitario.

Desde el punto de vista legal tenemos algunas referencias, como por ejemplo lo ejes que orientan la planificación de la formación universitaria: docencia, investigación, extensión y proyección social.

A nivel operativo, el esfuerzo sostenido ha sido la construcción de las referencias instrumentales: los factores, las variables, los indicadores y las fuentes de verificación, tanto en los términos cualitativos como cuantitativos, requiriendo un trabajo concertado y participativo de la comunidad universitaria.

\section{LOS CAMINOS HACIA LA MEJORA}

La autoevaluación es el trabajo técnico que realiza una institución para diagnosticar el estado que se encuentra en un momento determinado, cuyo objetivo inmediato es la mejora del funcionamiento institucional y cuya meta a mediano o largo plazo es lograr la acreditación, mediada por un conjunto de procesos políticos y técnicos.

Dos caminos se distinguen en esta etapa de construir y lograr la calidad: 1) La autoevaluación para la calidad, y 2) La autoevaluacion de la gestión orientada por la ISO.

La autoevaluación para la calidad es un proceso de critica y autocrítica de cómo funciona el sistema académico en la formación universitaria, tomando como referencia la concepción de universidad, la investigación, la construcción y/o reconstrucción de conocimientos, la formación profesional, la enseñanza, el aprendizaje y las competencias, buscando la mejora y la autorregulación. Tiene como tarea construir los indicadores mínimos de funcionamiento

La Autoevaluación de la gestión orientada por la ISO, es la evaluación de la satisfacción de los usuarios, a los que se les llama clientes, y tiene sustento en la normas de la familia ISO 9000.

Es muy conocida la guía IWA 2 «Aplicación de ISO 9001:2000 en educación», que establece determinadas condiciones sobre los procesos educativos, las responsabilidades de los directivos, en base a ciertas generalidades «técnicas», de cómo debería funcionar una institución educativa. Estos instrumentos surgieron en Inglaterra (Birmingham, 2001), donde se elaboró la Guía de la norma ISO 9000, posteriormente es aprobado y legalizado por el Comité Técnico 176, ISO 9001-2000, a través de un taller Internacional (Internacional Workshop Agreement IWA) y difundido en un documento titulado International Workshop Agrement 2 (IWA2), fijando como sede en Ginebra, con la tarea de actualizar las normas de calidad. El objetivo, establecer la regularidad de funcionamiento del modelo de la empresa de producción y de servicios.

Así tenemos que en la implementación de la gestión de calidad, el primer paso es promover la cultura de calidad, que son los principios difundidos por la ISO 9000, y después buscar la calidad, orientada por los siguientes principios: (1)La organización centrada en el alumno, (2) El liderazgo, (3) Involucrar a todo el personal y comprometerlo en hacer todo bien (4) El 
enfoque a procesos, (5) El enfoque a sistemas, (6) La mejora continua, (7) La toma de decisiones acertadas, y (8) las relaciones de mutuo beneficio.

\section{LA AUTOEVALUACIÓN PARA LOGRAR LA CALIDAD}

Es necesario aproximarnos al contenido y a las proyecciones de la calidad, para lo cual iniciamos citando la referencia de la UNESCO.

«La calidad ha llegado a ser una de las preocupaciones fundamentales de la educación superior. Ello obedece a que la satisfacción de las expectativas y las necesidades de la sociedad en relación con la educación superior depende, en última instancia de la calidad de su personal, sus programas y sus estudiantes, así como de su estructura y su entorno académico. La búsqueda de la «calidad» tiene múltiples facetas y el principal objetivo de las medidas tendientes a aumentar esa calidad deberá ser el automejoramiento tanto de sus instituciones como de todo el sistema» ${ }^{1}$.

De otro lado es necesario enfatizar que San Marcos ha sido la primera universidad pública que inició su autoevaluación en el año 2003. Su primer paso fue la publicación del documento titulado Lineamientos para una política de calidad, autoevaluación y acreditación ${ }^{2}$.

Desde el plano político, la autoevaluación requiere el compromiso de la comunidad universitaria, es decir la participación concertada de los estamentos involucrados en su funcionamiento. En segundo lugar, la autoevaluación es una reflexión y autoestudio realizado por la propia institución. En tercer lugar, la autoevaluación busca precisar la manera cómo está funcionando la universidad, estableciendo una distancia entre el modo de funcionamiento en el momento y aquello que sustenta su existencia. En cuarto lugar, la autoevaluación identifica errores e incoherencias, buscando corregir los desajustes hallados.

En cuanto a la acreditación, es una meta que se plantean alcanzar las universidades, en un proceso más o menos prolongado, y que involucra un conjunto de acciones, como lo podemos apreciar en el siguiente pensamiento:

«La acreditación de alta calidad no se refiere a las condiciones previas ni a requisitos básicos de desempeño; se refiere fundamentalmente a ver cómo una institución y sus programas se orientan hacia un deber ser, hacia un ideal de excelencia y pueden mostrar resultados específicos, tradición consolidada, impacto y reconocimiento social, entre otros. Mientras los estándares básicos (registro calificado) se constituyen en un primer escalón para ofrecer un servicio educativo de calidad, la acreditación se encuentra en el escalón siguiente puesto que supera las características mínimas de calidad y posee una dinámica orientada hacía la excelencia académica» ${ }^{3}$

\section{METODOLOGíA DE LA AUTOEVALUACIÓN}

Como hemos destacado anteriormente la autoevaluación requiere el compromiso y la identidad con la universidad que queremos mejorar, existiendo algunas fases que hay que

1 UNESCO (1995). Políticas para el cambio y el desarrollo de la educación superior, p. 2 ICFES.

2 Depaz Toledo, Enón y Cuba Sancho, uana (2003). Lineamientos para una política de calidad, autoevaluación y acreditación en la UNMSM: Lima OCCA.

3 CNA (2003). Lineamientos para la acreditación de programas, pp. 18 y 19. 
ejecutar y garantizar su continuidad, de una manera esquemática presentamos la primera fase, referida a la construcción de la matriz de autoevaluación para la carrera de psicología.

La propuesta que presentamos a continuación es el resultado de una investigación publicada anteriormente en esta revista ${ }^{4}$.

La matriz de autoevaluación universitaria para la carrera de psicología es una propuesta surgida a partir del compromiso de las autoridades que dirigen la facultad en el periodo 2004-2007. Esta iniciativa se realizó en mérito a cumplirse cincuenta años de la creación de la carrera de psicología en San Marcos y en el Perú. Como se recuerda el 27 de abril de 1955, se crea la sección de psicología en el Instituto de Filosofía de la antigua facultad de letras, aprobándose el primer plan de estudios. Cincuenta años después, el 27 de abril de 2005, mediante RR N ${ }^{\circ}$ 02136-R-05, se crea la unidad de calidad académica y acreditación (UCAA) de la facultad de psicología, aprobando la elección realizada por el Consejo de Facultad, del Mg. Diego Oswaldo Orellana Manrique como jefe de la mencionada unidad.

El Plan operativo 2006 de la UCAA define la estrategia de construir los estándares del funcionamiento académico de la EAP de Psicología, planteándose tres acciones: (1) Sensibilización sobre los objetivos del programa, (2) Autoevaluación de la calidad universitaria, y (3) Plan de mejora, que orientan cuatro objetivos generales y su doble como objetivos específicos. El primer objetivo general se propone «adaptar la matriz de autoevaluación propuesto por la OCCAA de la UNMSM.

Producto de dicha proyección presentamos la matriz de autoevaluación para que cumpla las fases y las acciones derivadas que conduzcan al Plan de mejora de la Facultad de Psicología.

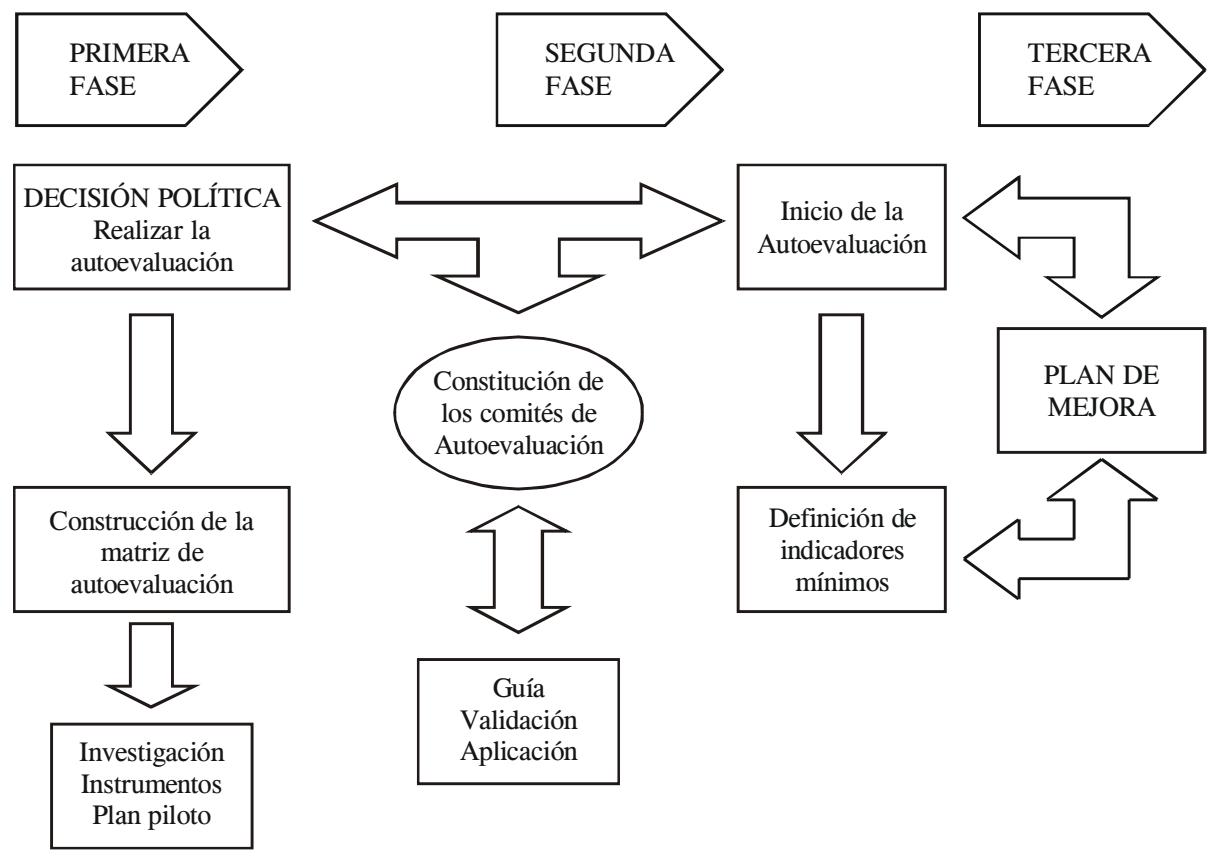

4 Orellana, Osaldo y Orellana Daphne (2005). Encuadre de un modelo alternativo para el estudio de la calidad universitaria. Una propuesta para psicología» En Revista de Investigación en Psicología, Vol 8, № Lima: UNMSM. 


\begin{tabular}{|c|c|c|c|}
\hline \multicolumn{4}{|c|}{ MATRIZ DE AUTOEVALUACIÓN UNIVERSITARIA PARA LA CARRERA DE PSICOLOGIA } \\
\hline \multicolumn{4}{|c|}{ (Referencia la UNMSM) } \\
\hline \multicolumn{4}{|c|}{ Factor 1: Proyecto de desarrollo universitario } \\
\hline $\mathbf{N}^{0}$ & VARIABLE & INDICADOR & FUENTE DE VERIFICACIÓN \\
\hline 1 & $\begin{array}{l}\text { Paradigma de } \\
\text { Universidad }\end{array}$ & $\begin{array}{l}\text { - Precisión conceptual del tipo de universidad, que se } \\
\text { esta manteniendo, defendiendo y/o construyendo, } \\
\text { deslindando con otros "modelos" ajenos a la } \\
\text { universidad, que no diferencian el carácter de ser } \\
\text { una institución de producción de conocimientos, } \\
\text { validación de los mismos (formación académica y } \\
\text { profesional) y debate de las utopias (filosofía del } \\
\text { desarrollo humano), y en cambio la reducen a la } \\
\text { oferta y la demanda del mercado } \\
\text { - Historia de los Planes estratégicos que han } \\
\text { orientado la planificación de la institución, desde su } \\
\text { fundación hasta la actualidad. } \\
\text { - Periodo de vigencia del actual Plan de Desarrollo } \\
\text { Universitario, o su similar: Plan Estratégico. }\end{array}$ & $\begin{array}{l}\text { - Plan de Desarrollo Universitario. } \\
\text { - Análisis de documentos del PDU. } \\
\text { - Resolución de decanato y Rectoral. } \\
\text { - Encuesta. }\end{array}$ \\
\hline 2 & $\begin{array}{l}\text { Visión y Misión } \\
\text { Universitaria }\end{array}$ & $\begin{array}{l}\text { - La visión y la misión es redactada de acuerdo al } \\
\text { consenso de la comunidad psicológica de la } \\
\text { universidad. } \\
\text { - En la visión se explicita la concepción de } \\
\text { universidad, la producción de conocimientos y la } \\
\text { formación profesional, dentro de un contexto de la } \\
\text { calidad universitaria. } \\
\text { - En la misión se explicita el compromiso de la } \\
\text { universidad con la búsqueda de la verdad, el } \\
\text { desarrollo nacional y el desarrollo humano, al } \\
\text { mismo tiempo su historia y las capacidades } \\
\text { sobresalientes. }\end{array}$ & $\begin{array}{l}\text { - Actas de redacción y debate de la visión y } \\
\text { misión universitaria. } \\
\text { - Plan Estratégico vigente. } \\
\text { - Publicación en texto y virtual en la página } \\
\text { WEB. } \\
\text { - Encuesta. }\end{array}$ \\
\hline 3 & $\begin{array}{l}\text { Presencia de la } \\
\text { Universidad en la } \\
\text { realidad nacional }\end{array}$ & $\begin{array}{l}\text { - } \begin{array}{l}\text { Convenios con instituciones de la comunidad y } \\
\text { organismos estatales y privados. }\end{array} \\
\text { - Participación en mesas de trabajo permanente, } \\
\text { convocadas por la sociedad civil. } \\
\text { - Intervención de la Facultad de Psicología en los } \\
\text { medios de comunicación. } \\
\text { - Reconocimiento público de instituciones de la } \\
\text { sociedad civil. }\end{array}$ & $\begin{array}{l}\text { - Resoluciones Decanales y Rectorales. } \\
\text { - Encuesta. }\end{array}$ \\
\hline 4. & $\begin{array}{l}\text { Logros obtenidos } \\
\text { Institucionalmente }\end{array}$ & $\begin{array}{l}\text { - Plan de desarrollo universitario o similar, producto } \\
\text { de la participación activa de la comunidad } \\
\text { psicológica de la universidad. } \\
\text { - Infraestructura moderna e implementada con } \\
\text { tecnología informática de punta, que favorece el } \\
\text { desarrollo académico y profesional. } \\
\text { - Premios nacionales e internacionales sobre la } \\
\text { formación académica y profesional. }\end{array}$ & $\begin{array}{l}\text { - Memorias anuales. } \\
\text { - Encuesta. }\end{array}$ \\
\hline 5 & $\begin{array}{l}\text { Participación en } \\
\text { redes } \\
\text { universitarias }\end{array}$ & $\begin{array}{l}\text { - Alianzas estratégicas con universidades peruanas } \\
\text { para mejorar la calidad en la formación de } \\
\text { psicólogos. } \\
\text { - Alianzas estratégicas universidades extranjeras } \\
\text { para llevar a cabo experiencias innovadoras en la } \\
\text { formación de psicólogos. }\end{array}$ & $\begin{array}{l}\text { - Documentos. } \\
\text { - Resolución del Decanato y Rectoral. }\end{array}$ \\
\hline 6 & $\begin{array}{l}\text { Organización de } \\
\text { eventos } \\
\text { (nacionales e } \\
\text { internacionales) }\end{array}$ & $\begin{array}{l}\text { - Organización de eventos científicos nacionales de } \\
\text { Psicología. } \\
\text { - Organización de eventos cientificos internacionales } \\
\text { de Psicología. }\end{array}$ & $\begin{array}{l}\text { - Documentos de convocatoria. Memoria de } \\
\text { eventos. } \\
\text { - Encuesta. }\end{array}$ \\
\hline
\end{tabular}




\begin{tabular}{|c|c|c|c|}
\hline \multicolumn{4}{|c|}{ Factor 2: Gobierno da la Facultad y Gestión } \\
\hline $\mathbf{N}^{\circ}$ & VARIABLE & INDICADOR & FUENTE DE VERIFICACIÓN \\
\hline 1 & $\begin{array}{l}\text { Consejo de Facultad } \\
\text { como órgano de } \\
\text { gobierno }\end{array}$ & $\begin{array}{l}\text { - Los miembros del consejo de facultad, elegidos de } \\
\text { acuerdo a las normas universitarias legales y conducidos } \\
\text { por un comité electoral autónomo. } \\
\text { - } \quad \text { Las autoridades de la facultad, elegidas democráticamente } \\
\text { por el consejo de facultad, de acuerdo a las normas } \\
\text { universitarias legales y conducidas por un comité } \\
\text { electoral autónomo. } \\
\text { - } \quad \text { Número de sesiones mensuales del consejo de facultad. } \\
\text { - } \quad \text { Asistencia de representantes de docentes, estudiantes y } \\
\text { - egresados. } \\
\text { - } \quad \text { Actas del consejo de facultad. } \\
\text { - Cumplimiento de acuerdos del Consejo de Facultad. }\end{array}$ & $\begin{array}{l}\text { - Citaciones. } \\
\text { - Actas publicadas, en texto y } \\
\text { virtual. } \\
\text { - Resoluciones Decanales y } \\
\text { Rectorales. }\end{array}$ \\
\hline 2. & $\begin{array}{l}\text { Composición del } \\
\text { consejo de facultad }\end{array}$ & $\begin{array}{l}\text { - } \text { Representación de docentes principales, asociados y } \\
\text { auxiliares. } \\
\text { - } \\
\text { - } \\
\text { Representación estudiantil. } \\
\text { Representación de egresados. }\end{array}$ & $\begin{array}{l}\text { - Actas del C.F. } \\
\text { - Resoluciones Decanales y } \\
\text { Rectorales. }\end{array}$ \\
\hline 3 & $\begin{array}{l}\text { Normas para la toma } \\
\text { de decisiones }\end{array}$ & $\begin{array}{ll}\text { - } & \text { Reglamento sobre el funcionamiento del Consejo de } \\
& \text { Facultad. } \\
\text { - } & \text { Lineamientos de política sobre el desarrollo universitario. } \\
\text { - } & \text { Lineamientos sobre el semestre y/o año académico. } \\
\text { - } & \text { Lineamientos sobre el funcionamiento administrativo. } \\
\text { - } \quad \text { Lineamientos sobre el mantenimiento de la infraestructura } \\
\text { - } \quad \text { la seguridad. }\end{array}$ & $\begin{array}{l}\text { - Resoluciones Decanales y } \\
\text { Rectorales. } \\
\text { - Encuestaa docentes, } \\
\text { estudiantes y administrativos. }\end{array}$ \\
\hline \multicolumn{4}{|c|}{ Factor 3: Producción de conocimientos } \\
\hline 1 & $\begin{array}{l}\text { Líneas de } \\
\text { investigación }\end{array}$ & $\begin{array}{l}\text { - Líneas de investigación explicitas y orientadoras de los } \\
\text { proyectos de investigación financiadas y no financiadas } \\
\text { por el consejo superior de investigación. }\end{array}$ & $\begin{array}{l}\text { - Documentos del II.Ps. } \\
\text { - Informe Anual de monitoreo. } \\
\text { - Encuestas. }\end{array}$ \\
\hline 2 & $\begin{array}{l}\text { Funcionamiento del } \\
\text { Instituto de } \\
\text { Investigaciones } \\
\text { Psicológicas }\end{array}$ & $\begin{array}{l}\text { - Directivos del Instituto de Investigaciones Psicológicas } \\
\text { elegidos mediante elecciones universales. } \\
\text { - Sesiones del Comité Directivo presidida por el Director del } \\
\text { instituto de investigaciones psicológicas. }\end{array}$ & $\begin{array}{l}\text { - Resoluciones Decanales y } \\
\text { Rectorales. } \\
\text { - Actas del Comité Directivo. }\end{array}$ \\
\hline 3 & $\begin{array}{l}\text { Docentes } \\
\text { investigadores }\end{array}$ & $\begin{array}{l}\text { - Número de docentes investigadores, clasificados como } \\
\text { miembros y colaboradores. } \\
\text { - Número de proyectos de investigación, financiados y no } \\
\text { financiados. por el consejo superior de investigación. }\end{array}$ & $\begin{array}{l}\text { - Resoluciones Decanales y } \\
\text { Rectorales. } \\
\text { - Actas de concurso anual de } \\
\text { proyectos. }\end{array}$ \\
\hline 4 & $\begin{array}{l}\text { Publicación de } \\
\text { Investigaciones }\end{array}$ & $\begin{array}{l}\text { - Número anual de publicaciones de la revista de } \\
\text { investigaciones psicológicas. } \\
\text { - } \quad \text { Número de investigaciones publicadas anualmente. }\end{array}$ & $\begin{array}{l}\text { - Revista de Investigaciones en } \\
\text { Psicología. } \\
\text { - Publicaciones de } \\
\text { investigaciones en otras } \\
\text { revistas. }\end{array}$ \\
\hline \multicolumn{4}{|c|}{ Factor 4: Normatividad de funcionamiento de la Facultad de Psicología y/o Escuela Académico Profesional } \\
\hline 1 & $\begin{array}{l}\text { Fundación y/o } \\
\text { creación de la EAP } \\
\text { y/o Facultad }\end{array}$ & $\begin{array}{l}\text { - Documentos que legalizan la fundación y/o creación de la } \\
\text { carrera de Psicología. } \\
\text { - Planteamiento inicial de objetivos fundacionales del } \\
\text { compromiso científico, cultural y ético. } \\
\text { - } \quad \text { Criterios fundacionales acordes con el momento histórico } \\
\text { y su contribución para superar el subdesarrollo y la } \\
\text { pobreza del país. } \\
\text { - Periodización de la historia de la formación profesional en } \\
\text { la universidad. }\end{array}$ & $\begin{array}{l}\text { - Actas de fundación. } \\
\text { - Resoluciones Decanales y/o } \\
\text { Rectorales. } \\
\text { - Encuesta. }\end{array}$ \\
\hline 2 & $\begin{array}{l}\text { Estructura Académico } \\
\text { Administrativa }\end{array}$ & $\begin{array}{l}\text { - } \quad \text { Sustento legal de la estructura académico-profesional. } \\
\text { - } \quad \text { Organigrama de la Facultad. } \\
\text { - } \quad \text { Cuadro de asignación de personal actualizado al año. } \\
\text { - } \quad \text { Manual de organización y funciones actualizado al año. } \\
\text { - } \quad \text { Comisión transitoria de actualización de las normas de } \\
\quad \text { funcionamiento universitario. }\end{array}$ & $\begin{array}{l}\text { - Ley Universitaria. } \\
\text { - Estatuto universitario. } \\
\text { - Publicación visual del } \\
\text { organigrama en texto y virtual. } \\
\text { - Resolución de aprobación del } \\
\text { CAP. } \\
\text { - Resoluciones Decanales y } \\
\text { Rectorales. }\end{array}$ \\
\hline 3. & $\begin{array}{l}\text { Reglamento y Manual } \\
\text { de procedimiento }\end{array}$ & $\begin{array}{l}\text { - } \quad \text { Manual de procedimientos actualizado y vigente. } \\
\text { - Comisión transitoria de actualización del manual de } \\
\text { procedimientos. }\end{array}$ & \\
\hline
\end{tabular}




\section{Factor 5: Perfiles docentes}

\begin{tabular}{|c|c|c|c|}
\hline 1 & $\begin{array}{l}\text { Cuadros } \\
\text { Académico } \\
\text { docentes } \\
\text { (grados y } \\
\text { títulos) }\end{array}$ & $\begin{array}{l}\text { - Perfil vigente de competencias docentes. } \\
\text { - Legajo personal actualizado. } \\
\text { - Capacitación continua a cargo del departamento académico. } \\
\text { - Estímulos a los docentes que aportan al desarrollo académico y } \\
\text { administrativo. } \\
\text { - Estadística docente con el nivel de formación alcanzado: grados } \\
\text { académicos y títulos profesionales. } \\
\text { - Carga docente semestral y anual. } \\
\text { - Premios y distinciones docentes. } \\
\text { - Intercambio docente. } \\
\text { - Evaluación del desempeño docente antes de culminar el semestre } \\
\text { académico. }\end{array}$ & $\begin{array}{l}\text { - Documento oficial del sistema } \\
\text { curricular. } \\
\text { - Plan de trabajo del departamento } \\
\text { académico de Psicología. } \\
\text { - Resoluciones Decanales y } \\
\text { Rectorales. } \\
\text { - Legajo personal. } \\
\text { - } \text { Carga académica. } \\
\text { - Informe de la EAP de Psicología. } \\
\text { - Encuesta. }\end{array}$ \\
\hline 2 & $\begin{array}{l}\text { Condición } \\
\text { laboral }\end{array}$ & $\begin{array}{l}\text { - Número de profesores nombrados y contratados. } \\
\text { - Convocatoria a concursos públicos. } \\
\text { - Evaluación para la promoción docente. } \\
\text { - Evaluación para la ratificación docente. }\end{array}$ & $\begin{array}{l}\text { - Reglamentos. } \\
\text { - Encuestas. }\end{array}$ \\
\hline 3 & $\begin{array}{l}\text { Categoría y } \\
\text { clase docente }\end{array}$ & $\begin{array}{l}\text { - Núm. de docentes por categoría y clase docente. } \\
\text { - Responsabilidad académica y administrativa de los profesores } \\
\text { principales. } \\
\text { - Responsabilidad académica y administrativa de los profesores } \\
\text { asociados. } \\
\text { - Carga docente-estudiantil. } \\
\text { - Número de docentes a dedicación exclusiva. } \\
\text { - Número de docentes a tiempo completo. } \\
\text { - Número de docentes a tiempo parcial. }\end{array}$ & $\begin{array}{l}\text { - Cuadros docentes del } \\
\text { departamento de psicología. } \\
\text { - Carga docente. } \\
\text { - Encuesta. }\end{array}$ \\
\hline 4 & $\begin{array}{l}\text { Organización } \\
\text { académica } \\
\text { docente } \\
\text { (secciones) }\end{array}$ & $\begin{array}{l}\text { - Estructuras académicas que organizan a los docentes para el } \\
\text { cultivo de la especialización en un área de la psicología (secciones } \\
\text { del departamento académico de psicología). }\end{array}$ & $\begin{array}{l}\text { - Actas de las estructuras } \\
\text { orgánicas docentes. } \\
\text { - Plan de trabajo del departamento } \\
\text { académico de psicología. }\end{array}$ \\
\hline 5 & $\begin{array}{l}\text { Producción } \\
\text { Intelectual }\end{array}$ & $\begin{array}{l}\text { - Publicaciones realizadas por los docentes: ediciones con ISBN y } \\
\text { registrados en la universidad (Consejo Superior de Investigación } \\
\text { UNMSM). } \\
\text { - Publicaciones registradas en base de datos del Sistema de } \\
\text { Bibliotecas de la universidad (SISBI / UNMSM). } \\
\text { - Publicaciones registradas en la base de datos de la Biblioteca } \\
\text { Nacional. }\end{array}$ & $\begin{array}{l}\text { - Textos publicados por docentes. } \\
\text { - Base de datos bibliográficos del } \\
\text { Sistema de Bibliotecas. } \\
\text { - Base de datos bibliográficos de la } \\
\text { Biblioteca Nacional. }\end{array}$ \\
\hline 6 & $\begin{array}{l}\text { Participación } \\
\text { anual en } \\
\text { eventos } \\
\text { académicos }\end{array}$ & $\begin{array}{l}\text { - Participación en eventos internos: análisis y valorización. } \\
\text { - Participación en eventos internacionales: análisis y valorización. } \\
\text { - Participación en eventos nacionales: análisis y valorización. }\end{array}$ & $\begin{array}{l}\text { - Legajo personal. } \\
\text { - Encuesta. }\end{array}$ \\
\hline \multicolumn{4}{|c|}{ Factor 6: Perfiles Estudiantiles } \\
\hline 1 & $\begin{array}{l}\text { Criterios de } \\
\text { admisión } \\
\text { universitaria }\end{array}$ & $\begin{array}{l}\text { - Normas anuales sobre la admisión a la EAP de Psicología. } \\
\text { - Cuadros de contenidos temáticos para el examen de admisión. } \\
\text { - Puntaje obtenido por ingresantes. } \\
\text { - Ubicación de la EAP de Psicología en el ranking de ingresantes. }\end{array}$ & $\begin{array}{l}\text { - Catalogo de admisión. } \\
\text { - Actas del Comité Asesor de la } \\
\text { EAP de Psicología. } \\
\text { - Informes estadísticos de la OCA. }\end{array}$ \\
\hline 2 & $\begin{array}{l}\text { Criterios de } \\
\text { permanencia }\end{array}$ & $\begin{array}{l}\text { - Guías de orientación al estudiante sobre la matrícula y la } \\
\text { progresión académica. } \\
\text { - Normas sobre evaluación del aprendizaje. } \\
\text { - Ciclos extraordinarios de verano que favorece la recuperación } \\
\text { académica. }\end{array}$ & $\begin{array}{l}\text { - Documentos en texto y virtual. } \\
\text { - Reglamentos. } \\
\text { - Normas especificas. }\end{array}$ \\
\hline 3 & $\begin{array}{l}\text { Perfiles de } \\
\text { competencias: } \\
\text { de ingreso, de } \\
\text { proceso y de } \\
\text { egreso }\end{array}$ & $\begin{array}{l}\text { - Perfil estudiantil de ingreso a la universidad adecuadamente } \\
\text { planteado. } \\
\text { - Perfiles de competencia de desarrollo progresivo. } \\
\text { - Perfil de competencias del egresado de Psicología. }\end{array}$ & $\begin{array}{l}\text { - Documentos técnicos. } \\
\text { - Encuesta. }\end{array}$ \\
\hline 4 & $\begin{array}{l}\text { Política de } \\
\text { estimulo a los } \\
\text { estudiantes }\end{array}$ & $\begin{array}{l}\text { - Lineamientos sobre estímulo al rendimiento académico. } \\
\text { - Modalidades de estímulos al rendimiento académico. } \\
\text { - Becas Estudiantiles. }\end{array}$ & $\begin{array}{l}\text { - Resoluciones Decanal y Rectoral. } \\
\text { - Documentos normativos. }\end{array}$ \\
\hline 5 & $\begin{array}{l}\text { Tiempo de } \\
\text { permanencia } \\
\text { para concluir } \\
\text { sus estudios }\end{array}$ & $\begin{array}{l}\text { - Permanencia de } 10 \text { ciclos académicos y dos ciclos de práctica } \\
\text { profesional (practicum / internado). } \\
\text { - Progresión académica por promociones o bases estudiantiles. }\end{array}$ & $\begin{array}{l}\text { - Informe de la Oficina de } \\
\text { matricula. }\end{array}$ \\
\hline
\end{tabular}




\begin{tabular}{|c|c|c|c|}
\hline \multicolumn{4}{|c|}{ Factor 7: Propuesta Curricular } \\
\hline 1 & $\begin{array}{l}\text { Fundamentación } \\
\text { académica } \\
\text { (Paradigmas y } \\
\text { áreas del } \\
\text { conocimiento) }\end{array}$ & $\begin{array}{l}\text { - Docentes y estudiantes identifican los paradigmas y las teorías } \\
\text { psicológicas presentes en la formación profesional. } \\
\text { - Conocimiento de los modelos epistemológicos que fundamentan la } \\
\text { cientificidad de la psicología. }\end{array}$ & $\begin{array}{l}\text { - Documentos curriculares. } \\
\text { - Encuesta. }\end{array}$ \\
\hline 2 & $\begin{array}{l}\text { Fundamentación } \\
\text { profesional del } \\
\text { Psicólogo }\end{array}$ & $\begin{array}{l}\text { - Documento que sustenta la formación académica y profesional del } \\
\text { psicólogo. } \\
\text { - Definición del psicólogo profesional y los ámbitos de trabajo } \\
\text { especializado. } \\
\text { - Plan de trabajo de la Escuela Académico Profesional de Psicologia. } \\
\text { - Definición del perfil de competencias de formación académico y } \\
\text { profesional, identificando, capacidades, destrezas y habilidades, así } \\
\text { como compromiso ético y formación moral. } \\
\end{array}$ & $\begin{array}{l}\text { - Documentos curriculares. } \\
\text { - Encuesta. }\end{array}$ \\
\hline 3 & $\begin{array}{l}\text { Sistema de } \\
\text { enseñanza }\end{array}$ & $\begin{array}{l}\text { - Definición de ejes transversales en la enseñanza-aprendizaje. } \\
\text { - Plan de trabajo del departamento académico sobre sistema de } \\
\text { enseñanza y monitoreo. } \\
\text { - Coordinación académica de la organización docente (secciones) para } \\
\text { el dictado de las asignaturas. } \\
\text { - Sílabos de asignaturas donde se explicite: objetivos de aprendizaje, } \\
\text { competencias, valores, metodologia, evaluación, cronograma y } \\
\text { referencias bibliográficas. }\end{array}$ & $\begin{array}{l}\text { - Documentos curriculares. } \\
\text { - Encuesta. }\end{array}$ \\
\hline 4 & $\begin{array}{l}\text { Duración de la } \\
\text { carrera }\end{array}$ & $\begin{array}{l}\text { - Organización de la formación profesional, de acuerdo a semestres y } \\
\text { años académicos que completen diez semestres de aprendizaje y dos } \\
\text { semestres de práctica preprofesional. } \\
\text { - Reglamento de matricula del estudiante regular. }\end{array}$ & $\begin{array}{l}\text { - Reglamento de matricula. } \\
\text { - Documentos curriculares. } \\
\text { - Encuesta. }\end{array}$ \\
\hline 5 & $\begin{array}{l}\text { Áreas } \\
\text { Curriculares }\end{array}$ & $\begin{array}{l}\text { - Correspondencia de las áreas cuniculares y las áreas disciplinarias de } \\
\text { la psicología. } \\
\text { - Áreas curriculares que promuevan la investigación. } \\
\text { - Áreas curriculares que promuevan el desarrollo personal. } \\
\end{array}$ & $\begin{array}{l}\text { - Documentos curriculares. } \\
\text { - Encuesta. }\end{array}$ \\
\hline 6 & $\begin{array}{l}\text { Clasificación de } \\
\text { las asignaturas }\end{array}$ & $\begin{array}{l}\text { - Criterios explícitos que sustentan la clasificación de las asignaturas. } \\
\text { - El papel de la interdisciplinariedad en la clasificación de las } \\
\text { asignaturas. }\end{array}$ & $\begin{array}{l}\text { - Documentos curriculares. } \\
\text { - Encuesta. }\end{array}$ \\
\hline 7 & Plan de Estudios & $\begin{array}{l}\text { - Plan de estudios debidamente estructurado, actualizado y vigente. } \\
\text { - Criterios de evaluación y reajuste del plan de estudios. } \\
\text { - Tabla de equivalencias y convalidaciones. } \\
\end{array}$ & $\begin{array}{l}\text { - Documentos curriculares. } \\
\text { - Encuesta. }\end{array}$ \\
\hline 8 & $\begin{array}{l}\text { Prácticas } \\
\text { preprofesionales }\end{array}$ & $\begin{array}{l}\text { - Funcionamiento de la oficina de prácticas preprofesionales. } \\
\text { - Número de docentes coordinadores de las prácticas preprofesionales. } \\
\text { - Sesiones de coordinación de las prácticas preprofesionales. } \\
\text { - Convenio con instituciones donde se realizan las prácticas } \\
\text { preprofesionales. } \\
\text { - Perfil de competencias del estudiante en el periodo de prácticas } \\
\text { preprofesionales. } \\
\text { - Criterios de evaluación del periodo de prácticas preprofesionales. }\end{array}$ & $\begin{array}{l}\text { - Documentos curriculares. } \\
\text { - Reglamento de prácticas } \\
\text { preprofesionales. } \\
\text { - Encuesta. }\end{array}$ \\
\hline 9 & $\begin{array}{l}\text { Vigencia del } \\
\text { actual currículo }\end{array}$ & \begin{tabular}{|l|} 
- Periodo de vigencia del currículo vigente. \\
- Criterios para la modificación y actualización del currículo vigente.
\end{tabular} & $\begin{array}{l}\text { - Documentos curriculares. } \\
\text { - Encuesta. }\end{array}$ \\
\hline \multicolumn{4}{|c|}{ Factor 8: Servicio de Bienestar y tutoría } \\
\hline 1 & $\begin{array}{l}\text { Programas de } \\
\text { Bienestar }\end{array}$ & $\begin{array}{l}\text { - Diagnóstico socioeconómico de los estudiantes. } \\
\text { - Atención a casos sociales que requieren ayuda. } \\
\text { - Programa de convivencia universitaria. }\end{array}$ & $\begin{array}{l}\text { - } \quad \text { Plan de trabajo de la } \\
\text { Dirección académica. } \\
\text { - } \quad \text { Encuesta. }\end{array}$ \\
\hline 2 & $\begin{array}{l}\text { Programas de } \\
\text { Orientación del } \\
\text { estudiante } \\
\end{array}$ & $\begin{array}{l}\text { - Funcionamiento de Oficina de servicio de asesoria, orientación y } \\
\text { tutoria al estudiante. } \\
\text { - Servicio de consejería especial. } \\
\end{array}$ & $\begin{array}{ll}\text { - } & \text { Resoluciones Decanales y } \\
& \text { Rectorales. } \\
\text { - } & \text { Reglamentos. } \\
\end{array}$ \\
\hline 3 & $\begin{array}{l}\text { Sistema de } \\
\text { Tutoría }\end{array}$ & $\begin{array}{l}\text { - Cuadros de docentes tutores para cada una de las promociones. } \\
\text { - Sistema de orientación de la tutoría. } \\
\text { - Eventos semestrales sobre la tutoría. }\end{array}$ & $\begin{array}{ll}\text { - } & \text { Carga académica. } \\
\text { - } & \text { Resoluciones Decanales y } \\
& \text { Rectorales. }\end{array}$ \\
\hline \multicolumn{4}{|c|}{ Factor 9: Equipamiento Técnico Pedagógico } \\
\hline 1 & $\begin{array}{l}\text { Infraestructura } \\
\text { Pedagógica } \\
\text { (Aulas) }\end{array}$ & $\begin{array}{l}\text { - Aulas con mobiliario adecuado que favorezca el estudio y el trabajo en } \\
\text { equipo. } \\
\text { - Aulas con equipos informáticos de última generación. }\end{array}$ & $\begin{array}{l}\text { - Plan de trabajo de la } \\
\text { Dirección Administrativa. } \\
\text { - Encuesta. }\end{array}$ \\
\hline 2 & $\begin{array}{l}\text { Laboratorios de } \\
\text { Psicologia } \\
\text { Experimental }\end{array}$ & $\begin{array}{l}\text { - Equipamiento de laboratorios con instrumental de última generación. } \\
\text { - Empleo cotidiano de laboratorios para el entrenamiento académico y } \\
\text { profesional. }\end{array}$ & $\begin{array}{l}\text { - Plan de trabajo de la EAP de } \\
\text { Psicología. } \\
\text { - Encuesta. }\end{array}$ \\
\hline 3 & $\begin{array}{l}\text { Gabinete de } \\
\text { Tests } \\
\text { Psicológicos }\end{array}$ & \begin{tabular}{ll|} 
- & Funcionamiento del gabinete de pruebas psicológicas. \\
- & Clasificación de las pruebas psicológicas. \\
- & Empleo de las pruebas psicológicas en el proceso de enseñanza \\
& aprendizaje. \\
\end{tabular} & $\begin{array}{l}\text { - Plan de trabajo de la EAP de } \\
\text { Psicología. } \\
\text { - Encuesta. }\end{array}$ \\
\hline
\end{tabular}




\begin{tabular}{|c|c|c|c|}
\hline \multicolumn{4}{|c|}{ Factor 10: Recursos bibliográficos } \\
\hline 1 & $\begin{array}{l}\text { Funcionamiento de } \\
\text { la biblioteca } \\
\text { especializada }\end{array}$ & $\begin{array}{l}\text { - Plan de trabajo de la biblioteca especializada. } \\
\text { - Organización y funcionamiento de la biblioteca. } \\
\text { - } \text { Boletín bibliográfico. }\end{array}$ & $\begin{array}{l}\text { - Plan de trabajo de la Dirección } \\
\text { Académica. } \\
\text { - Encuesta. }\end{array}$ \\
\hline 2 & $\begin{array}{l}\text { Ingreso anual de } \\
\text { titulos bibliográficos }\end{array}$ & - Estadistica sobre ingreso de títulos bibliográficos. & $\begin{array}{l}\text { - Plan de trabajo de la Dirección } \\
\text { Académica. } \\
\text { - Encuesta. }\end{array}$ \\
\hline 3 & $\begin{array}{l}\text { Ingreso anual de } \\
\text { revistas } \\
\text { especializadas }\end{array}$ & - Número de suscripciones a revistas especializadas. & $\begin{array}{l}\text { - Plan de trabajo de la Dirección } \\
\text { Académica. } \\
\text { - Encuesta. }\end{array}$ \\
\hline 4 & $\begin{array}{l}\text { Consulta } \\
\text { bibliográfica: } \\
\text { Presencial y virtual }\end{array}$ & - Soporte informático para la búsqueda bibliográfica. & $\begin{array}{l}\text { - Plan de trabajo de la Dirección } \\
\text { Académica. } \\
\text { - Encuesta. }\end{array}$ \\
\hline \multicolumn{4}{|c|}{ Factor 11: Soporte de nuevas tecnologías informáticas y comunicación } \\
\hline 1 & $\begin{array}{l}\text { Funcionamiento de } \\
\text { la oficina de } \\
\text { informática }\end{array}$ & - Organización y funciones de la Oficina de Informática. & $\begin{array}{l}\text { - Plan de trabajo de la Dirección } \\
\text { Administrativa. } \\
\text { - Encuesta. }\end{array}$ \\
\hline 2 & $\begin{array}{l}\text { Informatización de } \\
\text { los procesos de } \\
\text { matrícula }\end{array}$ & $\begin{array}{l}\text { - Informatización de la matrícula. } \\
\text { - Atención, asesoría y mantenimiento del soporte informático. }\end{array}$ & $\begin{array}{l}\text { - Plan de trabajo de la Dirección } \\
\text { Académica. } \\
\text { - Encuesta. }\end{array}$ \\
\hline 3 & $\begin{array}{l}\text { Informatización de } \\
\text { actas de calificación } \\
\text { de asignaturas }\end{array}$ & $\begin{array}{l}\text { - Informatización del llenado de actas de calificación de } \\
\text { asignaturas. }\end{array}$ & $\begin{array}{l}\text { - Plan de trabajo de la Dirección } \\
\text { Académica. } \\
\text { - Encuesta. }\end{array}$ \\
\hline 4 & $\begin{array}{l}\text { Informatización de } \\
\text { la consulta } \\
\text { bibliográfica }\end{array}$ & - Boletines virtuales sobre actualización bibliográfica. & $\begin{array}{l}\text { - Plan de trabajo de la Dirección } \\
\text { Académica. } \\
\text { - } \text { Encuesta. }\end{array}$ \\
\hline 5 & $\begin{array}{l}\text { Funcionamiento de } \\
\text { la enseñanza virtual }\end{array}$ & $\begin{array}{l}\text { - Empleo de plataformas virtuales haciendo uso de las TICs en la } \\
\text { enseñanza de las asignaturas del Plan de Estudios. } \\
\text { - Experiencias del empleo de plataformas virtuales de } \\
\text { asignaturas e-learning. }\end{array}$ & $\begin{array}{l}\text { - Plan de trabajo de la Dirección } \\
\text { Académica. } \\
\text { - Encuesta. }\end{array}$ \\
\hline 6 & $\begin{array}{l}\text { Equipamiento para } \\
\text { teleconferencias }\end{array}$ & - Equipo de teleconferencia. & - Equipos. \\
\hline \multicolumn{4}{|c|}{ Factor 12: Grados y Títulos } \\
\hline 1 & Grado de Bachiller & $\begin{array}{l}\text { - Perfil de competencias básicas para optar el grado académico } \\
\text { de Bachiller. } \\
\text { - Tiempo entre la culminación de estudios y optar el grado } \\
\text { académico de Bachiller. } \\
\text { - Número de alumnos por modalidades para optar el grado } \\
\text { académico de Bachiller. }\end{array}$ & $\begin{array}{l}\text { - Plan de trabajo de la Dirección } \\
\text { Académica. } \\
\text { - Encuesta. }\end{array}$ \\
\hline 2 & Titulo de Psicólogo & $\begin{array}{l}\text { - Perfil de competencias especializadas para optar el título } \\
\text { profesional de psicólogo. } \\
\text { - Tiempo entre la culminación de estudios y optar el título } \\
\text { profesional de psicólogo. } \\
\text { - Número de alumnos por modalidades para optar el título } \\
\text { profesional de psicólogo. }\end{array}$ & $\begin{array}{l}\text { - Plan de trabajo de la Dirección } \\
\text { Académica. } \\
\text { - Encuesta. }\end{array}$ \\
\hline \multicolumn{4}{|c|}{ Factor 13: Egresados } \\
\hline 1 & $\begin{array}{l}\text { Registro de } \\
\text { Egresados }\end{array}$ & $\begin{array}{l}\text { - Libro de registro de egresados. } \\
\text { - Organización de los egresados de Psicología. } \\
\text { - Participación de los egresados en la elección de su } \\
\text { representante al Consejo de Facultad. }\end{array}$ & $\begin{array}{l}\text { - Plan de trabajo de la Dirección } \\
\text { de la EAP de Psicología. } \\
\text { - Encuesta. }\end{array}$ \\
\hline 2. & $\begin{array}{l}\text { Protagonismo en el } \\
\text { campo } \\
\text { especializado }\end{array}$ & - Cuadros de egresados que destacan en el campo profesional. & $\begin{array}{l}\text { - Plan de trabajo de la Dirección } \\
\text { de la EAP de Psicología. } \\
\text { - Encuesta. }\end{array}$ \\
\hline 3 & $\begin{array}{l}\text { Protagonismo en la } \\
\text { política }\end{array}$ & - Cuadros de egresados que destacan en el campo político. & $\begin{array}{l}\text { - Plan de trabajo de la Dirección } \\
\text { de la EAP de Psicología. } \\
\text { - Encuesta. }\end{array}$ \\
\hline 4 & $\begin{array}{l}\text { Mercado laboral } \\
\text { (perfil ocupacional) }\end{array}$ & $\begin{array}{l}\text { - Requerimientos de instituciones públicas y privadas de } \\
\text { psicólogos egresados de la universidad. }\end{array}$ & $\begin{array}{l}\text { - Plan de trabajo de la Dirección } \\
\text { de la EAP de Psicología. } \\
\text { - Encuesta. }\end{array}$ \\
\hline
\end{tabular}




\begin{tabular}{|c|c|c|c|}
\hline \multicolumn{4}{|c|}{ Factor 14: Red orgánica universitaria a la que pertenece } \\
\hline 1 & $\begin{array}{l}\text { Comunicación con } \\
\text { universidades } \\
\text { extranjeras }\end{array}$ & $\begin{array}{l}\text { - Convenios de cooperación internacional para el intercambio } \\
\text { de docentes y estudiantes } \\
\text { - Participación en proyectos conjuntos de carácter disciplinar e } \\
\text { interdisciplinario }\end{array}$ & $\begin{array}{l}\text { - Resoluciones Decanales y } \\
\text { Rectorales. } \\
\text { - Encuesta. }\end{array}$ \\
\hline 2 & $\begin{array}{l}\text { Comunicación con } \\
\text { universidades } \\
\text { nacionales }\end{array}$ & $\begin{array}{l}\text { - Intercambio de experiencias universitarias de formación } \\
\text { profesional con universidades peruanas }\end{array}$ & $\begin{array}{ll}\text { - } & \text { Resoluciones Decanales y } \\
& \text { Rectorales. } \\
\text { - } & \text { Encuesta. }\end{array}$ \\
\hline 3 & $\begin{array}{l}\text { Sistema de elección } \\
\text { del par externo }\end{array}$ & $\begin{array}{l}\text { - Mecanismos de elección de par externo con objetivos de } \\
\text { mejora de la calidad }\end{array}$ & $\begin{array}{ll}\text { - } & \text { Resoluciones Decanales y } \\
& \text { Rectorales. } \\
\text { - Encuesta. } & \\
\end{array}$ \\
\hline \multicolumn{4}{|c|}{ Factor 15: Apoyo Administrativo } \\
\hline 1 & $\begin{array}{l}\text { Funcionamiento } \\
\text { administrativo }\end{array}$ & $\begin{array}{l}\text { - Estructura orgánica administrativa que favorece el } \\
\text { funcionamiento académico } \\
\text { - Perfil del personal administrativo idoneo para el apoyo } \\
\text { académico docente y estudiantil. }\end{array}$ & $\begin{array}{l}\text { - Plan de trabajo de la Dirección } \\
\text { administrativa. } \\
\text { - Encuesta. }\end{array}$ \\
\hline 2 & $\begin{array}{l}\text { Manual de } \\
\text { procedimientos } \\
\text { administrativos }\end{array}$ & $\begin{array}{l}\text { - Manual de procedimientos actualizado que simplifica los } \\
\text { trámites administrativos }\end{array}$ & $\begin{array}{ll}\text { - } & \text { Resoluciones Decanales y } \\
& \text { Rectorales. } \\
\text { - } & \text { Encuesta. } \\
\text { - } & \text { Resultados de encuestas. } \\
\end{array}$ \\
\hline \multicolumn{4}{|c|}{ Factor 16 : Infraestructura } \\
\hline 1 & $\begin{array}{l}\text { Edificación } \\
\text { especializada }\end{array}$ & $\begin{array}{l}\text { - Local edificado para el funcionamiento de la formación } \\
\text { académica y profesional }\end{array}$ & \multirow[t]{3}{*}{$\begin{array}{l}\text { - } \text { Resoluciones Decanales y } \\
\text { Rectorales. } \\
\text { - Encuesta. }\end{array}$} \\
\hline 2 & $\begin{array}{l}\text { Aulas y } \\
\text { equipamiento }\end{array}$ & $\begin{array}{l}\text { - Aulas construidas con adecuada iluminación y recursos } \\
\text { didácticos y espacio requerido para la instrucción universitaria. }\end{array}$ & \\
\hline 3 & $\begin{array}{l}\text { Laboratorio y } \\
\text { equipamiento }\end{array}$ & $\begin{array}{l}\text { - Ambientes de laboratorio construidos para el entrenamiento } \\
\text { académico del método experimental. }\end{array}$ & \\
\hline 4 & $\begin{array}{l}\text { Biblioteca } \\
\text { especializada }\end{array}$ & $\begin{array}{l}\text { - Ambiente construido para la biblioteca con características } \\
\text { especializadas para su óptimo funcionamiento. }\end{array}$ & \\
\hline \multicolumn{4}{|c|}{ Factor 17: Extensión universitaria y proyección social } \\
\hline 1 & $\begin{array}{l}\text { Promoción del } \\
\text { Psicólogo } \\
\text { profesional : } \\
\text { publicaciones y } \\
\text { eventos }\end{array}$ & $\begin{array}{l}\text { - Difusión de la imagen del psicólogo en distintos ambientes de } \\
\text { la población y empleando distintos medios: presenciales y } \\
\text { virtuales. }\end{array}$ & $\begin{array}{l}\text { - Plan de trabajo del CEUPS. } \\
\text { - Encuesta. }\end{array}$ \\
\hline 2 & $\begin{array}{l}\text { Promoción de la } \\
\text { Psicología como } \\
\text { conocimiento } \\
\text { especializado: } \\
\text { Eventos }\end{array}$ & $\begin{array}{l}\text { - Participación en equipos multidisciplinarios con profesionales } \\
\text { de distintas áreas que intervienen en la población }\end{array}$ & $\begin{array}{l}\text { - Plan de trabajo del CEUPS. } \\
\text { - Encuesta. }\end{array}$ \\
\hline 3 & $\begin{array}{l}\text { Centros comunales } \\
\text { de docencia y } \\
\text { entrenamiento } \\
\text { profesional }\end{array}$ & $\begin{array}{l}\text { - Convenios con instituciones de la comunidad. } \\
\text { - Convenios con instituciones del Estado. } \\
\text { - Centro de docencia para el entrenamiento profesional. } \\
\text { - Proyectos de desarrollo comunitarios. }\end{array}$ & $\begin{array}{l}\text { - Plan de trabajo del CEUPS. } \\
\text { - Encuesta. }\end{array}$ \\
\hline 4 & $\begin{array}{l}\text { Cursos permanentes } \\
\text { de extensión }\end{array}$ & $\begin{array}{l}\text { - Organización de eventos de capacitación dirigidos a } \\
\text { psicólogos y disciplinas afines. }\end{array}$ & $\begin{array}{l}\text { - Plan de trabajo del CEUPS. } \\
\text { - Encuesta. }\end{array}$ \\
\hline \multicolumn{4}{|c|}{ Factor 18: Presupuesto } \\
\hline 1 & Presupuesto anual & $\begin{array}{l}\text { - Presupuesto anual consensuado y aprobado por el Consejo } \\
\text { de Facultad. }\end{array}$ & $\begin{array}{l}\text { - Plan de trabajo de la Dirección } \\
\text { Administrativa. } \\
\text { - Encuesta. }\end{array}$ \\
\hline 2 & $\begin{array}{l}\text { Inversión por } \\
\text { estudiante }\end{array}$ & - Índices de inversión anual por estudiante. & $\begin{array}{l}\text { - Plan de trabajo de la Dirección } \\
\text { Administrativa. } \\
\text { - Encuesta. }\end{array}$ \\
\hline 3 & Ingresos brutos & - Registro anual de ingresos a la facultad por recursos propios. & $\begin{array}{l}\text { - Plan de trabajo de la Dirección } \\
\text { Administrativa. } \\
\text { - Encuesta. }\end{array}$ \\
\hline 4 & Egresos & - Registro de egresos de la facultad. & $\begin{array}{l}\text { - Plan de trabajo de la Dirección } \\
\text { Administrativa. } \\
\text { - Encuesta. }\end{array}$ \\
\hline
\end{tabular}




\begin{tabular}{|c|c|c|c|}
\hline \multicolumn{4}{|c|}{ Factor 19: Proyectos innovadores } \\
\hline 1 & Planes de mejora & $\begin{array}{l}\text { - Proyectos de mejora de carácter innovador presentados por } \\
\text { docentes, estudiantes y/o egresados. }\end{array}$ & $\begin{array}{l}\text { - } \quad \text { Plan de trabajo de la Dirección } \\
\text { Académica. } \\
\text { - } \quad \text { Plan de trabajo del } \\
\text { departamento académico. } \\
\text { - } \quad \text { Encuesta. }\end{array}$ \\
\hline \multicolumn{4}{|c|}{ Factor 20: Proyecciones para el futuro } \\
\hline 1 & $\begin{array}{l}\text { Para el desarrollo de } \\
\text { la formación } \\
\text { profesional }\end{array}$ & $\begin{array}{l}\text { - Identificación de los paradigmas internacionales de la } \\
\text { formación profesional del psicólogo. } \\
\text { - Planteamiento de soluciones en respuesta a desafíos, riesgos } \\
\text { e incertidumbres. }\end{array}$ & $\begin{array}{l}\text { - } \text { Proyectos. } \\
\text { - } \text { Encuestas. }\end{array}$ \\
\hline 2 & $\begin{array}{l}\text { Para el desarrollo de } \\
\text { la investigación }\end{array}$ & - Proyectos de apertura de áreas del conocimiento. & $\begin{array}{l}\text { - Plan de trabajo del I.I.Ps. } \\
\text { - Encuesta. }\end{array}$ \\
\hline
\end{tabular}

\section{REFERENCIAS BIBLIOGRÁFICAS}

1. AI (2004). Acuerdo institucional 2001-2006 : Balance y propuesta Lima : UNMSM

2. Alarcón, Reynaldo (2000). Historia de la Psicología en el Perú de la colonia a la república. Lima: URP

3. Ausubel, David y otros (1995). Psicología Educativa. Un punto de vista cognoscitivo México:Trillas

4. Arnaz, José (1993). La planeación curricular México: Trillas

5. Bruner, J. Goodnow J. YAustin G (19780) El proceso mental en el aprendizaje. Madrid: Narcea.

6. Díaz Barriga, Ágel (1992). Ensayos sobre problemática curricular México:Trillas

7. Diaz Barriga, Frida y otros (1990). Metodología del diseño curricular para educación superior México: Trillas

8. Facultad de Psicología (2001). Gacetilla Psicológica. Lima: Fac Psicología UNMSM

9. EAP (1995). Currícula de pregrado para la formación de Psicólogo Lima: UNMSM

10. Gimeno Sacristán, José (1989). El currículum una reflexión sobre la práctica Madrid:Morata

11. Ernández, Pedro (1995). Diseñar y enseñar. Teoría y técnicas de la programación del proyecto docente Barcelona: NARCEA

12. IIPS (1998). Catalogo de investigaciones. Lima: IIPSIC UNMSM

13. IIPS (1998) Memorias Taller de investigación en Psicología. Políticas y prioridades Lima: IIPS. UNMSM

14. OCCAA (2005). Hacia la autoevaluación en San Marcos. Lima: UNMSM

15. Orellana, O y Orellana D (2005). Encuadre de un modelo alternativo para el estudio de la calidad universitaria. Una propuesta para psicología. En Revista de investigación en psicología Vol $8 \mathrm{~N}^{\boldsymbol{o}} 2$ Lima: UNMSM

16. Rectorado (2003). Transparencia y fortalecimiento institucional: Mensaje a la comunidad universitaria. Lima: UNMSM

17. Titone, Renzo (1981) Psicodidáctica Madrid: Narcea

18. UNMSM-OGP (2005). Compendio estadístico 2004. Lima, UNMSM 